\title{
Schädigungen des Auges durch Licht. Senile Linsentrübungen und senile Maculadegeneration.
}

\author{
Von \\ Prof. J. van der Hoeve, \\ Leiden.
}

Natürliche und artifizielle Lichtquellen können Schädigungen am Auge hervorrufen, wenn sie in ungewöhnlicher Stärke kürzere oder längere Zeit auf das Sehorgan einwirken: Nach Hertel können alle Strahlen das Auge schädigen, wenn ihre Intensität nur genügend ist.

Von den Abschnitten des Spektrums, welche die Schädigungen verursachen können, sind die ultraroten Strahlen noch am wenigsten in dieser Beziehung studiert, wahrscheinlich spielen sie, wie Vogt meint, eine bedeutende Rolle bei der Entstehung des Glasbläserstares; den leuchtenden Strahlen wird allgemein der größte Einfluß beim Auftreten der Sonnenblendung zugeschrieben, während die Wirkung der ultravioletten Strahlen auf das Auge am besten untersucht ist.

Bei Schädigungen des Auges durch ultraviolettes Licht ist es immerhin sehr schwer, die Beteiligung von leuchtenden Strahlen auszuschlieBen, so daß wir am besten tun, wenn wir reden über die Wirkung von sehr viele ultraviolette Strahlen enthaltendem Licht.

Wenn wir in den Referaten von $v . H e \beta^{1}$ ) und $P$ arsons ${ }^{2}$ ) und bei Wagenmann ${ }^{3}$ ) nachsehen, welche Teile des Auges durch die Einwirkung dieser Strahlen geschädigt werden können, so bemerken wir, daß fast kein einziger Teil des Auges davor geschützt ist.

Nicht alle Strahlen niedriger Wellenlänge, welche auf das Auge fallen, dringen ins Innere desselben ein; die Media lassen nicht alle durch.

Obwohl die Resultate der Untersuchungen von Brücke, Donders und $v$. Rees, de Chardonnet, Widmark, Schulek, Hertel, Schanz und Stockhausen, Birch-Hirschfeld, Hallauer, Vogt, Parsons und Henderson, Martin u. a. nicht in allen Teilen uber-

1) C. v. He B, Über Schädigungen des Auges durch Licht. Intern. Congress of Medicine, London 1913.

2) H. W. Parsons, Affections of the eye produced by undue exposure to light. Intern. Congress of Medicine, London 1913.

3) Wagenmann, Die Verletzungen des Auges durch ultraviolette Strahlen. Graefe-Saemisch, Handbuch d. G. A. 9, 5. Abt., S. 1683.

v. Graefes Archiv 1. Ophthalmologie. Bd. 98. 
einstimmend sind, so können wir daraus doch mit Bestimmtheit entnehmen, daß Hornhaut und Linse die ultravioletten Strahlen in bedeutendem Maße aufhalten.

Die Hornhant hält alle Strahlen von weniger als ungefähr $300 \mu \mu$ (nach Martin als $295 \mu \mu$ ) beinahe vollständig auf, während Strahlen von höherer. Wellenlänge nach Martin nicht absorbiert werden.

Nach demselben Untersucher absorbiert die Linse alle Strahlen von kürzerer Wellenlänge als $350 \mu \mu$ absolut, von $400-350 \mu \mu$ partiell. Versuche von Halla uer, Birch-Hirschfeld, Schanz und Stockha usen u. a. haben aber gezeigt, daß diese Absorption durch die Linse abhängt von Lebensalter, Konstitution und weiteren noch nicht genügend festgestellten individuellen Verhältnissen (Dicke, Färbung und Konsistenz der Linse), wodurch die Wellenlänge und Zahl der durchgelassenen ultravioletten Strahlen individuell außerordentlich verschieden ist. Im jugendlichen Alter gelangen nach Halla uer noch von den Strahlen von $310-330 \mu \mu$ ein mehr oder weniger großer Teil zur Netzhaut, während auch Schanz eine jugendliche Linse untersuchte, welche ultraviolette Strahlen bis etwa $300 \mu \mu$ durchließ.

Die versehiedenen Autoren schreiben über die Durehlässigkeit der Linse für ultraviolette Strahlen immer, daß die Linse so viele dieser Strahlen absorbiert. Dies ist nicht ganz richtig, nur ein Teil des Mangels an Durchlässigkeit wird durch wirkliche Absorption erklärt. Die Linse kann die Strahlen am Durehtritt in verschiedener Weise verhindern, nämlich durch Reflexion, Fluorescenz, diffuse Zerstreuung $\left.{ }^{1}\right)$ und Absorption im engeren Sinn; wir müssen uns also vergegenwärtigen, daß, wenn geschrieben wird über die Absorption der ultravioletten Strahlen durch die Linse, damit meistens alle vier Einflüsse gemeint werden, welche den Strahlen den Durchtritt verhindern.

Von den ultravioletten Strahlen, welche auf unser Auge fallen, wirken also auf die äußeren Augenteile alle Strahlen ein:

auf Iris und Linse die Strahlen von $300-400 \mu \mu$,

auf die Netzhaut nur Strahlen von $310-400 \mu \mu$, meistens nur wenige in individuell sehr verschiedener Menge.

Um zu wissen, welche Sehädigungen Licht mit viel ultravioletten Strahlen dem Auge zuzuziehen vermag, kann man zwei Wegen folgen:

1. aus der Literatur heraussuchen, welche Schädigungen am menschlichen Auge durch derartiges Licht wahrgenommen sind;

2. experimentell an Tieren untersuchen, welche Schädigungen Bestrahlung mit viel ultraviolette Strahlen enthaltendem Licht an Tieraugen verursacht.

1) van der Hoeve, Ned. Tijdschr. voor Geneesk. 1912, S. 660, und Die optische Heterogenitat der Linse, dieses Archiv 98, 39. 1918. 
Wenn wir dies tun, so sehen wir, daß fast kein einziger Teil des Auges vor der Beschädigung sicher ist.

Durch natürliche Lichtquellen mit ultravioletten Strahlen werden. beim Menschen Augenabweichungen wahrgenommen bei der sog. Schneeblindheit, welche von ungefähr allen Autoren als durch Licht mit viel ultravioletten Strahlen verursacht betrachtet wird. Als kïnstliche Lichtquellen, welche derartige Schädigungen verursachen, finden wir: Quecksilberdampflampen, Bogenlampen, geschmolzenes Metall bei den elektrischen Wellen, Kurzschluß u. dgl. vermeldet, welche alle Ophthalmia electrica verursachen können; die Schädigungen durch Blitz werden; mehr als durch andere Ursachen erweckt, betrachtet. Den Typus der Schädigungen durch ultraviolette Strahlen enthaltendes Licht am Auge verursacht, gibt uns die Schneeblindheit, von welcher wir bei Wagenmann (S. 1675) die folgende Beschreibung lesen.

„Bei Schneeblendung kommen zwei verschiedene Gruppen von Veränderungen am Auge vor. Bei der am häufigsten beobachteten ersten Form handelt es sich um das Auftreten einer akuten, schmerzhaften. mit starken Reizerscheinungen einhergehenden Entzündung der Augenoberfläche. Brennender Schmerz oder lebhaftess Fremdkörpergefühl, starke Lichtscheu, Tränenträufeln und heftiger Lidkrampf, so daß die Augen nicht geöffnet werden können. Der mit sich steigernden Schmerzen einhergehende Reizzustand macht die davon Befallenen hilflos. Die Bindehaut erscheint gerötet und chemotisch, am stärksten im Lidspaltbereich. Es besteht Ciliarinjektion, manchmal spontaner Ciliarschmerz, ebenso Ciliardruckempfindlichkeit. Die Schmerzen können unerträglich werden." - Es kann zu Hornhautgeschwüren kommen, Pupillen stark verengt, Iris zuweilen verfärbt, Hyperämie der Papille und Netzhautgefäße.

Die zweite Gruppe von Augenveränderungen besteht aus Störungen der Netzhautfunktion. Nebenbèr bestehen Verdunklung des Gesichtsfeldes, bisweilen Nyctalopio oder Hemeralopie, Erythropsie, Zentralfarbenskotom wie bei Haenel; Anästhesie der Netzhaut mit Gesichtsfeldeinengung $u$. dgl.

Diese zwei Gruppen werden wahrscheinlich durch verschiedene Strahlen verursacht; die äußeren Abweichungen durch die ultravioletten Strahlen niedriger Wellenlänge, die Veränderung an der Netzhaut durch ultraviolette Strablen höherer Wellenlänge und Lichtstrahlen; hierdurch wird auch erklärt, daß jede für sich allein oder kombiniert auftreten kann, je nachdem das licht nur ultraviolett höherer oder nur niedriger Wellenlänge enthält oder beide.

Die Entzündungserscheinungen am Auge, welche hierbei entstehen, werden durch Parsons sehr charakteristisch Photophthalmie genannt, welche nach ihm typiert ist durch intensive "Photophobie: 
und Tränenfluß mit reflektorem Blepharospasmus und Ciliarneuralgien; akute Conjunctivitis und in den ernsteren Fällen Komplikationen von Hornhaut und Iris.

Die obengenannten artifiziellen Lichtquellen geben die gleichen Erscheinungen wie die Schneeblindheit. Wir finden erwähnt:

Lichtscheu - Tränenträufeln, Ciliarneuralgien.

An den Augenlidern: Erythem - Spasmus - Ödem.

An der Bindehaut: Epitheländerungen, Hyperämie, Ödem, Chemosis, Ekchymosen, Injektion, Entzündung.

Hornhaut: Trübungen, Infiltration, Ulceration.

Iris: Hyperämie, Verfärbung, Kontraktion der Pupille, Entzündung.

Corpus ciliare: Ciliardruckempfindlichkeit.

Papille: Hyperämie, Fntzündung, Abblassung.

Netzhaut: Skotome, besonders Zentralskotome, wie die Selbstbeobachtung Haenels bei Schneeblindheit, die Mitteilungen von Hertel durch Bogenlampe, von Birch-Hirschfeld durch Quecksilberdampflampenbestrahlung; nach Parsons ist bei Kurzschlußblendung gewöhnlich Zentralskotom anwesend; Ha ab nahm, ebenso wie Uh th of kleine graugelbe Herde in der Foveagegend wahr, Lubinski einen hellen Fleck in der Macula, andere, wie Schirmer, Gefäßabweichungen.

Bei dem zweiten Weg, sich Einsicht in die durch Licht mit viel ultravioletten Strahlen hervorgerufenen Schädigungen zu schaffen, der experimentellen Bestrahlung, finden wir verzeichnet, daß bei den vielen Versuchen von Widmark, HeB, Birch-Hirschfeld, Martin u.a. an Bindehaut und Hornhaut dieselben Änderungen wahrgenommen werden als die obengenannten und an der Iris Entzündung; überdies fand Birch-Hirschfeld am hinteren Epithel der Iris Quellung, Auflockerung und Anpressung an die Linse, sowohl bei pigmentierten Kaninchen wie bei albinotischen. An der Linse wurden Trübungen und Epithelveränderungen gefunden, welche bei vèrschiedenen Tieren aber immer nur im Pupillarteil der Linse auftraten. An den Ciliarfortsätzen Hyperämie, Blutungen, Quellung und Desquamation des Epithels, Entzündung mit fibrinösen Exsudaten in vorderer und hinterer Augenkammer, welche Entzündung an diesem Augenteil nach Birch Hirschfeld ganz besonders ausgeprägt war; in der Chorioidea Hyperämie und Blutungen; an der Netzhaut Degenerationen, besonders wenn die Augen linsenlos sind.

Wir sehen also, daß alle Teile des Auges bei Experimenten Schädigungen erleiden können durch heftige, relativ kurzdauernde Bestrahlung mit viel ultraviolette Strahlen enthaltendem Licht, und daß auch in der Natur und durch artifizielle Lichtquellen bisweilen dieselben Schädigungen verursacht werden. 
Ganz etwas anderes aber ist die Frage, ob das fortwährend auf das Auge einwirkende Licht mit ultravioletten Strahlen imstande ist, demselben Schaden zuzufügen.

Im diffusen Tageslicht kommen viele ultraviolette Strahlen vor, genügend, wie Schanz zeigte, um in unseren Linsen so starke Fluorescenz zu erregen, daß wir dieselbe in Schanz' Kästchen wahrnehmen können.

In fast allen unseren künstlichen Lichtquellen, vor allem in den neueren, sind viele ultraviolette Strahlen anwesend.

Also werden unsere Augen fortwährend, wenigstens solange sie geöffnet sind, durch Licht mit vielen ultravioletten Strahlen (Sonnenlicht, diffuses Tageslicht, artifizielle Lichtquellen) getroffen, und es ist nicht wunderzunehmen, daß gefürchtet wird, dies könnte die Augen schädigen.

Hertel und Henker behaupten, daß diffuses Tageslicht für die Augen unschädlich ist; so schreiben diese Autoren „,das sicher unschädliche Tageslicht"1), während sie Schanz und Stoekhausen vorwerfen ${ }^{2}$ ), daß dieselben über Schneeblindheit usw. schreiben als durch Tageslicht verursacht; während nach $H$. und $H$. ,durch das diffuse Tageslicht, sei es das Licht des blauen Himmels oder reflektiertes Wolkenlicht, oder Licht, das durch den bedeekten Himmel an trüben Tagen zu uns kommt, diese Affektionen sicher nicht hervorgerufen werden".

Sie nehmen also als Axiom an: das diffuse Tageslicht ist unschädlich.

Dieses Axiom ist aber mit später bekannt gewordenen Tatsachen im Streit, denn 1917 teilt Judson Daland ${ }^{3}$ ) mit, daß die Eskimos in Alaska, Sibirien und auf den Inseln des Beringmeers und im Arktischen Ozean sehr viel zu leiden haben von Schneeblindheit, und nicht nur an sonnigen Tagen, sondern auch an ",wolkigen und trüben Tagen"4). Diese ganz unentwickelten Leute machen sich selbst dagegen Schutzbrillen, welche sie auch an wolkigen Tagen benutzen. Mancher Alaskapionier ist Schlachtopfer geworden von dem Glauben, daß man an trüben Tagen ohne Schutzbrille umhergehen kann. Hierdurch ist also bewiesen, daß diffuses Tageslicht den Augen direkt schädlich sein kann.

Abet auch, wenn dem nicht so wäre und wirklich das diffuse Tageslicht in der Menge, worin unser Auge daran exponiert ist, nicht schadete, so folgt hieraus noch nicht, daß, wie Voege und Hertel und Henker meinen, jetzt die idealen Lichtquellen diejenigen sind, deren Ticht dem

\footnotetext{
1) Archiv 1. Ophthalmol. 76, Heft 1, \$. 214. 1910.

2) Archiv f. Ophthalmol. 73, Heft 3, S. 597. 1910.

8) Fiskimo-Snow Blindness and Goggles. Ophthalmic Record 3, 116. 1917.

4) Spatierung von Judson Daland.
} 
diffusen Tageslicht gleichkommt; ideale Schutzgläser diejenigen, welche starke Lichtquellen so dämpfen, daß die durchgelassene Helligkeit die Helligkeit diffus beleuchteter Wolken nicht übertrifft ${ }^{1}$ ).

Wenn z. B. im Winter der Tag acht Stunden dauert und wir arbeiten weitere acht Stunden bei einer dergleichen Ideallampe, so setzen wir unsere Augen zweimal so lange dieser Lichtmenge aus, als die Natur es tut, und es ist ganz gut möglich, daß eine tagtäglich wiederbolte lange Aussetzung des Auges an diesem Lichte dem Auge schadet, während eine kürzere Aussetzung das nicht tun würde.

Wie dem auch sei, die Beobachtung der Eskimos hat schon genügend bewiesen, daß die Ansicht, diffuses Tageslicht sei dem Auge unschädlich, irrig ist, deshalb können wir auch nicht als Axiom annehmen, daß Lichtquellen und Schutzgläser, welche das Licht dem diffusen Tageslicht gleich machen, ideale sind.

Wenn also Tageslicht die Augen schädigen kann, ist die weitere Frage, welche Schädigung es verursachen kann.

Bei den Alaskzeskimos fand Dr. D. S. Neuman aus Nonne, Alaska, bei den von Schneeblindheit geplagten Patienten sehr oft Chorioiditis und Retinitis, welehe nach Judson Daland durch die ultravioletten Strahlen verursacht sein können.

Bei diesen Eskimos nennt Judson Daland als vielfach vorkommende Augenkrankheiten Conjunctivitis, Katarakt, Trachom und Schneeblindheit. Die Conjunctivitis und Schneeblindheit können den ultravioletten Strahlen zugeschrieben werden. Eine weitere Frage, welche mehrfach aufgeworfen wurde, ist, ob die Entstehung der Katarakt, mit Namen der Alterstar, mit der Wirkung des Tageslichtes auf das Auge in Zusammenhang gebracht werden könne.

Wenn wir nachschlagen, was über diese Frage in der neueren Literatur vermeldet ist, so finden wir, daß Hirschberg') 1898 mitteilt, „die meisten staroperierten Hindus, die ich in den Krankenhäusern von Kalkutta, Jáipore, Bombay vorfand oder gelegentlich ermittelte, waren ungefähr um das 40 . Lebensjahr oder bald danach operiert worden, während bei uns das durchsehnittliche Alter für die Ausziehung des sogenannten Alterstars auf das 62. Lebensjahr fällt. Unter der glühenden Sonne von Indien reift also der Alterstar 20 Jahre früher als bei uns. In ähnlicher Weise ist auch bei unseren Landarbeitern die vollständige Trübung der Linse weit häufiger schon um das 50. Jahr zu beobachten als bei sonst gesunden Städtern."

Diese Angaben werden gewissermaßen bestätigt durch Schwitz.er and. Sehulek.

1) Hertel und Henker, 1. c. S. 610.

a) Über den Star der Glasbläser. Centralbl. f. prakt. Augenheilk. 1898, S. 115. 
Schwitzer ${ }^{1}$ ) entnimmt aus der Statistik über 3764 Fälle seniler Katarakte, die in den Jahren 1895-1896 an der Budapester Universitäts-Augenklinik zur Aufnahme und Behandlung gelangten, daß senile Katarakt hauptsächlich bei Feldarbeitern vorkommt, und zwar besonders bei solchen, die in der Ebene wohnen; er meint, diese Daten liefern den Beweis, daß die Sonnenstrahlen, und zwar die hypervioletten Strahlen, eine wesentliche Rolle in der Ätiologie der Cataracta senilis spielen.

Schulek ${ }^{2}$ ) bespricht eine außerordentlich interessante Statistik von Friedrich Groß aus dem Jahre 1857, aus welcher hervorgeht, daß bei Feldarbeit in den schattenlosen Ebenen Ungarns Star vielfach vorkommat. Wo die Frauen im Felde mitarbeiten, fand Groß bei Männèrn und Frauen den Star in gleicher Menge, während wo die Frauen sich nicht mit Feldarbeit beschäftigen, viel weniger Frauen starkrank sind als Männer. Schulek mißt dieser Arbeit von Groß, welche er bahnbrechend nennt, großen Wert bei und sieht in der Arbeit Schwitzers eine Bestätigung von Groß' Ansichten, welche Schulek teilt. Obwohl nach Hirschberg die Wärme der Sonne den größten Einfluß auf die Entstehnng des Stars ausübt, meint Schulek, daß man aus Hirschbergs Beobachtungen mit mehr Recht den Licht- und ultravioletten Strahlen eine Rolle darin beimessen muß.

Gegen oben mitgeteilte Ansichten treten Birch-Hirschfeld, Snell und $\mathrm{HeB}$ auf.

Birch-Hirschfeld ${ }^{3}$ ) bemängelt mit vollem Recht Schwitzers Arbeit, weil seine Literaturangaben unrichtig sind und seine Meinung, daß der senile Star durch die ultravioletten Strahlen des Sonnenlichtes entsteht, weil Widmark mit ultravioletten Strahlen Linsentrübungen verursachen konnte, ohne jeden Beweis ist. Diese Bemerkungen sind ganz richtig, aber daraus ergibt sich nicht, daß die unter Schuleks Auspizien angefertigte Statistik unrichtig ist, welche ergab, daß in Ungarn der Star am meisten vorkommt bei Feldarbeitern in den Ebenen.

Weiter führt Birch-Hirschfeld, nach v. Heß mit Recht, an, daß, wenn Schuleks u. a. Ansichten über das Entstehen des senilen Stars durch ultraviolette Strahlen des Tageslichtes richtig wären, der Star vorzugsweise solche Leute befallen müßte, welche den ultravioletten Strablen des Tageslichtes besonders ausgesetzt sind, wie die Bewohner von hochgelegenen Orten usw., dies ist jedoch, soweit bekannt, nicht der Fall.

Dieser Einwurf ist aber nicht richtig, in den hochgelegenen Orten

1) Sohwitzer, Beiträge zur Entstehung des grauen Alterstars. Ungarische Beiträge d. Augenheilk. 2, 291. 1900.

$\left.{ }^{2}\right)$ Schulek, Schutzbrillen gegen Ultraviolett usw. Ungarische Beiträge d. Augenheilk. 2, 467. 1900.

3) 1. C. S. 555 . 
kommen mehr ultraviolette Strahlen im Licht vor, weil die Zerstreuung in die reinere Atmosphäre nicht so stark ist, weil jedoch die Zerstreung der vierten Potenz der Wellenlänge umgekehrt proportional ist, kommt dies hauptsächlich den ultravioletten Strahlen niederer Wellenlänge zugute. Diese sind es, welche Irritation und Entzündung der äußeren Augenteile erregen, weshalb die Augen bei ihrer Zunahme mehr geschlossen gehalten oder geschützt werden, wodurch die Strahlen auf die Linse weniger einwirken können.

In eben gelegenen Gegenden, wo das Licht weniger ultraviolette Strahlen niedriger Wellenlänge enthält, aber wo durch stark reflektierende Flächen die Augen durch viele Strahlen getroffen werden, z. B. in den Polargegenden, scheint der Star auffallend viel vorzukommen, denn Judson Daland nennt bei den Eskimos Alaskas und Sibiriens Star eine „,common ocular disease".

Gegen Hirschbergs indische Erfahrung führt v. Heß an, daß S nell sagt: „Der Star tritt in Indien nicht früher auf als bei uns." Snell entnimmt dies den Angaben von Brockman.

Wenn wir aber Snells') Arbeit im Original lesen, so kann man darin den Beweis für diese Äußerung nicht finden.

Snell einigt sich mit Hirschberg über die Zeit, in welcher in Europa die Patienten zur Kataraktextraktion kommen, in Deutschland ist das nach Hirschberg im 62. Jahr; dies trifft nach Snell in seiner Praxis selbst. für Glasarbeiter zu. Von Brockman erhielt S nell die folgende Statistik über das Alter, in welchem in Indien von ihm Star operiert worden war.

$\begin{array}{cccr}\text { Alter } & \text { Männer } & \text { Weiber } & \text { total } \\ 21-30 & 99 & 59 & 158 \\ 31-40 & 309 & 298 & 607 \\ 41-50 & 872 & 893 & 1765 \\ 51-60 & 1275 & 762 & 1987 \\ 61-70 & 503 & 168 & 671\end{array}$

Die meisten Extraktionen fallen demnach auf das 41.-60. Jahr, also um das 50. Jahr; vergleichen wir diese Zahlen mit Snells Statistik von operierten Staren aus seiner Praxis in England und lassen wir das Alter von 21-30 Jahren fort, weil dies nicht zu den senilen Staren gerechnet werden kann, so sehen wir:

\begin{tabular}{|c|c|c|c|c|}
\hline \multirow[b]{2}{*}{$31-40$} & \multicolumn{2}{|c|}{ Brockman (Indien) } & \multicolumn{2}{|c|}{ Snell (England). } \\
\hline & 607 & $12 \%$ & 47 & $9 \%$ \\
\hline $41-50$ & 1765 & $35 \%]_{75}$ & 67 & $13 \%$ \\
\hline $51-60$ & 1987 & $40 \%\}^{70}$ & 159 & $81 \%$ \\
\hline $61-70$ & 671 & $13 \%$ & 237 & $47 \%$ \\
\hline & $\overline{5030}$ & & 510 & \\
\hline
\end{tabular}

1) Simeon Snell, An inquiry into the alleged frequency of Cataract in bottheraakers. Brit. med. Journ. 1907, S. 8. 
Wir sehen also, daß nach Snells Statistik das Operationsalter für $78 \%$ zwischen 50 und 70 Jahren liegt, nach Brockman in Indien für $75 \%$ zwischen 40 und 60 und daß bei $S$ nell im zweiten Dezennium dieses Zeitabschnittes die Operationen dem ersten viel weiter überlegen sind als in Brockmans Statistik, so daß man aus $\mathrm{Snells} \mathrm{Mitteilungen}$ nur entnehmen kann, daß in der Gegend in Indien, wo Brockman operierte, der Star mehr als zehn Jahre früher zur Operation kommt als in der Gegend Englands, wo Snell arbeitet. ISnell versucht dies zu erklären durch das schnellere Wachstum und frühere Ableben der Indier, aber das Faktum bleibt, woraus folgt, daß Snell - Brockmans Statistik statt einen Widerspruch eine Bestätigung von Hirschbergs Angabe ergibt. Auch der bekannte Staroperateur Coll. Smith äußert sich dabin, daß Star in Indien früher reift als in Europa $a^{1}$ ), so daß dieser Punkt wohl als endgültig erledigt betrachtet werden kann.

In der jüngsten Zeit finde ich eine Mitteilung von Fred G. Walter ${ }^{2}$ ), daß Stare frequenter werden, je mehr man sich dem Äquator nähert, und daß bewiesen ist, daß Licht ein Faktor ist, um dies zu verursachen. Die Abhandlung, in welcher Walter diesen Beweis liefert: (Paper by the author before the Florida Medical Association at the Land 1915) ist mir lejder nicht zugänglich. - Ascher ${ }^{3}$ ) teilt aus der Straßburger und Prager Universitäts-Augenklinik mit, daß er bei Patienten, bei denen das eine Auge stärker als das andere an grauem Star erkrankt war, ermitteln konnte, daß sie an Arbeitsplätzen gearbeitet hatten, wo das Licht auf das stärker erkrankte Auge mehr einzuwirken vermochte als auf das andere. - Wir sehen also, daß, während die Einwürfe von Birch - Hirschfeld nicht zutreffend sind, die genauen, ausführlichen und interessanten Angaben GroB', welche durch Schwitzer und. Schulek bestätigt werden, zeigen, daß in Ungarn der Star bei Landarbeitern, besonders in der Ebene, mehr auftritt als bei Städtern, daß Hirschberg mitteilt, wie in seiner Gegend Landarbeiter früher Star zeigen als Städter, daß nach Hirschberg in Indien der Star um das 40., nach Brockmans Statistik um das 50. Lebensjahr operiert wird, während in Deutschland und England nach Hirschberg und Snell dieses Alter etwa 62 ist; daß nach Judson Daland Star in den Schnee- und Eisgegenden Alaskas ein „,common disease“ ist, daß nach Walter für Amerika gilt: je näher der Äquator, je frequenter

1) La Clinique Ophtalmologique 1914.

2) Ophthalmic Record, Mareh 1917, S. 146.

s) Ascher, Über den Einfluß der Lage der lichtquelle auf die primäre Lokalisation des Alterstars. Med. Klin. 1917, Nr. 19. Zit. nach Wochenschr. . Therapie u. Hygiene d. Auges und nach Scha nz, v. Graefes Archiv f. Ophthalmol. 96, 175 . 
der Star, und daB nach Ascher die Stelle des Arbeitslichtes bestimmen kann, welches Auge am meisten an Star erkrankt.

Aus den bis jetzt in der Literatur niedergelegten Mitteilungen kommt man demnach zu dem Ergebnis, daß in Ländern und Gegenden, welche dem reflektierten Sonnenlicht, oder besser dem Licht mit viel ultraviolettèn Strahlen, mehr und länger exponiert sind, der Star besonders bei Personen, welche sich dem Lichte am meisten aussetzen, häufiger ist und früher auftritt als an anderen Orten.

Immerhin muß màn natürlich bedenken, daB Lichteinwirkung nicht die einzige Ursache des Stares sein wird, aber eine von den vielen, welche auf den Gesamtorganismus oder das Auge einwirken.

Stammten die obengenannten Mitteilungen aus einer einzigen Gegend, so könnte man meinen, daß Diät oder Wohnungsverhältnisse, Rasseneigenschaften usw. die Ursachen der häufigeren und früheren Starerscheinung wären, weil aber die Mitteilungen aus allen Gegenden der Welt: Alaska, Sibirien, Indien, Ungarn, Berlin, Florida stammen, können dergleichen Verhältnisse nur wenig Einfluß üben, und man kommt dazu, einen Grund, welcher allen diesen Gegenden gemeinsam ist, in casu die Anwesenheit vieler ultravioletter Strahlen, als Ursache anzunehmen.

Wie wir gesehen haben, sind die Gegenbeweise von Birch - Hirschfeld und S nell nicht zutreffend, bleibt noch die Äußerung von v. He ${ }^{1}{ }^{1}$, welcher schreibt:

„Aber auch aus allgemein biologischen Gründen könnte ich mich nicht leicht $z u$ der Annahme entschließen, daß an einem lebenden Gebilde, das sich phylogenetisch unter dauernder Wirkung des Tageslichtes entwickelt hat, die unzweckmäßige Eigentümlichkeit zur Ausbildung gekommen sein sollte, durch ebendieses Tageslicht so schwer geschädigt zu werden."

Dies ist eine persönliche Meinung, wofür keine Beweise geliefert werden und welche meines Erachtens nicht unangreifbar ist.

Es ist doch schwer zu sagen, was Tageslicht ist; für unsere Gegenden ist es gewöhnlich diffuses, vom Boden relativ wenig reflektiertes Licht, für die Eskimos, welche von Geschlecht auf Geschlecht in Schnee und Eisumgebung leben, ist es stark reflektiertes, abwechselnd diffuses und Sonnenlicht, am Aquator Sonnenlicht, und von den beiden letzten wissen wir durch Schnee- und Sonnenblendung, daß sie die Augen schädigen können.

Eine der Hauptursachen, welche machen kann, daß Organe unter EinfluB von fortwährend einwirkenden Schädlichkeiten wenig leiden, ist der Umstand, daß die am besten dagegen Widerstand leistenden Individuen die besten Lebensumstände haben und dadurch Geschlech-

1) 1. e. S. 154 . 
ter entstehen, welche von den Schädlichkeiten nicht oder nur wenig angegriffen werden: ,the survival of the fittest". - Dieser kräftige Faktor kommt bei der senilen Starbildung wenig in Betracht, weil der Star erst Beschwerde verursacht in einem Alter, worin das Individuum selten noch Kinder zeugt und worin überdies seine Arbeitskraft meistens schon geschädigt ist. Eine natürliche Selektion wird demzufolge hier nicht stattfinden.

Also, obwohl man $\vee$. HeB völlig beipflichten muB, daß, ,eine sorgfältige aus einheitlichen Gesichtspunkten durchgeführte Statistik über die relative Häufigkeit des Alterstars in verschiedenen Ländern in vieler Hinsicht von großem Interesse sein wird und voraussichtlich auf die in Rede stehende Frage Antwort geben kann $\left.^{1}\right)^{\prime c}$, weisen die bis jetzt in dieser Hinsicht bekannten Tatsachen darauf hin, daß der Alterstar frequenter und früher auftritt in Gegenden und Ländern und bei Leuten, welche dem Licht mit viel ultravioletten Strahlen am meisten exponiert sind.

Wenn der Einfluß ron Lichteinwirkung auf Starbildung wahrscheinlich ist, so ist eine weitere Frage, in welcher Weise Licht mit vielen ultravioletten Strahlen die Linse schädigen kann.

Bei den Experimenten von Widmark, Birch-Hirschfeld, v. HeB, Martin über die Wirkung von ultravioletten Strahlen gelang es in einigen Fällen, Degenerationen des Kapselepithels und Linsentrübungen zu erzengen; dieselben blieben aber immer auf den Pupillarteil der Linse beschränkt. Bei anderen Versuchen von Birch - Hirschfeld, Marrtin, Hertel, Ogneff, Strebel gelang es nicht, irgendwelche Veränderungen an der Linse hervorzurufen. Die erzeugten Trübungen zeigen keine Übereinstimmung mit dem Alterstar, welcher gewöhnlich nicht im Pupillarteil anfängt, und beweisen nur, daß ebenso als Bindehaut- und Hornhautepithel auch das Linsenepithel direkt geschädigt werden kann durch relativ kurzdauernde Bestrahlung mit außerordentlich kräftigen ultravioletten Strahlen.

Chardonnet und Widmark meinen beide, der bestrahlte Teil der Linse muß durch die Absorption Änderungen erleiden, auch diese würden dann im bestrahlten Pupillarteil ansetzen müssen. Schanz meint, die Bestrahlung setzt die Linseneiweißstoffe um von leichtlöslichen in schwerer lösliche, und sieht hierin die Ursache des senilen Stares; um zu erklären, daß auch außerhalb des Pupillarteils die Linse sich dabei trüben kann, benutzt Schanz die von mir angegebene diffuse Zerstreuung in der Linse. Wenn dies auch möglich ist, so würde doch die erste Wirkung im bestrahlten Linsenteil sich entfalten müssen, also im Pupillarteil, was bekanntlich bei dem senilen Star nicht der Fall ist.

x) He B, l, c. S. 154 . 
Jeder Versuch, die Entstehung von senilen Linsentrübungen durch direkte . Finwirkung von leuchtenden oder ultravioletten. Strahlen zu erklären, scheitert an dem Faktum, daß dieselben im Pupillarteil angreifen, der senile Star nicht dort anfängt.

Wir müssen also, um eine Verbindung zwischen senilem Star und Lichteinwirkung erklären zu können, neben der bei den Experimenten aafgefundenen direkten Linsenschädigung eine indirekte Sehädigung annehmen. Hierbei lenken sich unsere Gedanken sofört auf den Ciliarkörper, welcher für die Ernährung der Linse sorgt; 1912 habe ich angegeben, wie der Ciliarkörper bei Bestrahlung geschädigt. werden kann. - Daß er geschädigt wird, ist ein Faktum, welches bewiesen wird durch die Ciliardruckempfindlichkeit bei Schneeblindheit und vielleicht durch die bisweilen außerordentlich heftigen Ciliarneuralgien, welche bei Schneeblindheit und Ophthalmia electrica vorkommen, aber für alles durch Birch - Hirschfelds Versuche, wobei die Entzündungserscheinungen an den Ciliarfortsätzen ganz besonders ausgeprägt waren $^{1}$ ).

Wie ist es möglich, daß der Ciliarkörper durch Bestrahlung nicht nur Schaden erleidet, aber daß selbst die dadurch verursachte Entzündung der Ciliarfortsätze gegenüber den Abweichungen an anderen Teilen ganz besonders ausgeprägt genannt werden muß? Wir wissen doch, daß von allen Teilen des Auges die Ciliarfortsätze vor Bestrahlungen am besten geschützt erscheinen; von vorn durch Hornhaut und Iris, von der Seite durch Conjunctiva, Sclera, den Ciliarmuskel und Pigmentschicht, so daß Fuchs ${ }^{2}$ ) schreibt: Der Ciliarkörper wird weder selbst vom Lichte getroffen, noch verändert er sich irgendwie bei Lichteinwirkung und kommt dabei nicht in Frage."

Die einzige Seite, von welcher das sezernierende Ciliarepithel absolut unbeschützt daliegt, ist die innere Seite, und die optische Heterogenität der Linse macht es durch diffuse Zerstreuung möglich, daß es von dieser Seite durch die ins Auge fallenden Strahlen angegriffen wird. In früheren Mitteilungen ${ }^{3}$ ) habe ich gezeigt, wie die Linse einen Teil des Lichtes nach allen Seiten hin zerstreut und wohl die ultravioletten. Strahlen sehr bedeutend stärker als die leuchtenden Strahlen. Die hier in Anmerkung kommenden ultravioletten Strahlen von 300-400 $\mu \mu$ Wellenlänge, werden 16-50 mal so stark zerstreut als die roten Strahlen von $800 ; 2,4-7,7 \mathrm{mal}$ so stark als blaue Strahlen von $500 \mu \mu$ Wellenlänge. Weil die diffuse Zerstreuung in der Linse eine sehr deutliche Luminescenz ergibt, muß die Menge der zerstreuten ultravioletten Strahlen

1) 1. .. S. 509 .

2) Lehrbuch der Angenheilkunde, 12. Anfl. 1910, S. 22.

s) Ned. Tijdschr. voor Geneesk. 1912, S. 660; v. Greefes Archiv f. Ophthalmol. 1918. 
sehr bedeutend sein. - Diese Zerstreuung wird am stärksten sein in dem vorderen Linsenteil, weil mehr nach hinten zu der Gehalt an ultravioletten Strahlen schon bedeutend vermindert sein wird.

Das zerstreute Licht wird nach allen Seiten hingeworfen, und dadurch werden also am meisten die Wände der hinteren Augenkammer getroffen werden. Die hintere Fläche der Iris leidet bei der Bestrahlung, wie wir bei Birch-Hirschfeld ${ }^{1}$ ) lesen, deutlich: „Die Zellen des hinteren Pigmentbelags der Iris waren in einigen Fällen deutlich verändert. Sie erschienen im Querschnitt verbreitert, unregelmäßig gestaltet, von ihrer Unterlage teilweise aufgehoben und der vorderen Linsenkapsel angepreßt usw." Auch die Ciliarfortsätze werden einen großen Teil der zerstreuten Strahlen empfangen, während die vorderen Teile der Netzhaut und Aderhaut obwohl in viel geringerer Intensität auch zerstreute Strahlen bekommen, genügend wahrscheinlich, um die starke Hyperämie im vorderen Abschnitt der Aderhaut zu erklären.

Die Processi ciliares werden durch dieses viel ultraviolette Strahlen enthaltende Licht getroffen an ihrem sezernierenden Epithel, wodurch bei akuter heftiger Bestrahlung, wie in Birch-Hirschfelds Versuchen, die Veränderungen auftreten können: Hyperämie, Infiltration, fibrinöse Exsusdation, Quellung und beginnende Desquamation des Epithels. Durch das viel weniger intensive Licht, das die Augen den ganzen Tag hindurch trifft (diffuses Tageslicht, Sonnenlicht, artifizielle Lichtquellen), werden die Ciliarfortsätze nicht bis zur Entzündung gereizt, aber es ist sehr wahrscheinlich, daß diese fortwährende Reizung im Laufe der Jahre und Dezennien doch nicht ohne Folgen bleibt, so wie nach $\mathbf{F u c h s}{ }^{2}$ ) dis ultravioletten Strahlen bei weniger starker Einwirkung auch leichtere Veränderungen chronischer Art hervorrufen zu können scheinen. - Diese fortwährende Reizung braucht die Epithelien nur wenig zu schädigen, um derartige Änderungen des Sekretionsproduktes zu veranlassen, daß die Ernährung der Linse Schaden leidet.

In völligem Einklang mit dieser von $\operatorname{mir}^{3}$ ) 1912 mitgeteilten Hypothese ist das Resultat der Experimente von Henderson-Parsons und E. K. Martin ${ }^{4}$, daß Bestrahlung mit ultravioletten Strahlen die Funktion der Ciliarfortsätze ändert, so daß Hämolysine übertraten ins Kammerwasser von immunisierten Kaninchen und Meersehweinchen nach Bestrahlung mit Kromayers Lampe.

Wenn dem so ist, so ist es nicht unwahrscheinlich, daß der fortgesetzte Reiz des Ciliarepithels, welcher mit den Jahren zunimmt,

1) 1. c, S. 508 .

2) Fuchs, 1. o. S. 24.

3) Ned. Tijdschr, voor Geneesk. 1912, S. 660 .

4) Nach Parsons Referat London' 1913, S. 209. 
in Dezennien zu derartigen Ernährungsstörungen der Linse führt, daß Trübungen auftreten. Dieselben brauchen nicht in der Pupille anzufangen, aber werden dort auftreten, wo die Ernährung am meisten Schaden gelitten hat oder ein präformierter Locus minoris resistentiae anwesend ist. Wird die Bestrahlung zu stark, so können durch direkte Schädigung des Linsenepithels Trübungen verursacht werden, wie bei den Experimenten von Widmark, v. He B u. a. der Fall war; hierbei ist es nicht unmöglich, daB solche im Pupillarteil der Linse anfangende Trübungen sich darüber hinaus ausdehnen können, gleichzeitig mit oder ohne Trübungen an anderer Stelle.

In dieser Weise kann also ohne Mühe das Auftreten von senilem Star mit Anfang der Trübung außerhalb der Pupille erklärt wercden und es leuchtet auch ein, wie fast bei jedem Menschen in hohem Alter Linsentrübungen auftreten, und daß bei Leuten, welche lange Zeit der Einwirkung von viel Licht mit vielen ultravioletten Strahlen ausgesetzt sind, der Star eher reift als bei anderen Leuten, so daß die Beobachtungen von Hirschberg, Groß, Schulek, Schwitzer, Brockman, Judson Daland, Walter u. a. leicht erklärt werden.

Eine Folge dieser Hypothese ist, daß Linsen, welche die Netzhaut gegen Strahlen niedriger Wellenlänge gut schützen durch starke diffuse Zerstreuung, das Ciliarepithel am meisten schädigen und dadurch sich selbe am ersten trüben werden, während Linsen, welche wenig heterogen sind, die Strahlen niedriger Wellenlänge viel mehr durchlassen werden und also die Netzhaut nicht schützen, aber demgegenüber das Ciliarepithel nicht schädigen und also selber durchsichtig bleiben werden.

In dieser Beziehung würde die Ideallinse diejenige sein, welche so viel zerstreut; $d a ß$ sie die Netzhaut genügend schützt, und doch so wenig, dab das Ciliarepithel imstande bleibt, die Linse so zu ernähren, daß keine Trübungen auftreten.

Bekanntlich meint Peters, daß viele Stare von Schädigung des Ciliarepithels abhängen, und er konnte dieselbe öfters mikroskopisch nachweisen.

Daß diese Änderungen nicht immer mikroskopisch nachweisbar sind, ist nicht zu wundern, denn schon äußerst geringfügige. Sohädigungen des Epithels werden dergleichen Änderungen der die Linse ernährenden Flüssigkeiten verursachen können, daß die Linse jm Laufe der Jahre Schaden erleidet und sich trübt.

Wenn durch diffuse Zerstreuung der Ciliarkörper leidet, die Linse sich trübt, wird auch die hintere Irisschicht vor allem an dem Pupillarrand von ultravioletten Strahlen überschwemmt werden. Die hintere Irisschicht allein durch die Zerstreuung, der Pupillarrand von vorn. durch direkte Bestrahlung, von hinten durch die von der Linsenoberfläche reflektierten Strahlen und durch diffus zerstreute Strahlen. 
Obwohl diese Teile wahrscheinlich viel mehr Widerstand leisten können als das sezernierende Ciliarepithel, läßt sich doch erwarten, daB im Laufe der Jahre auch an diesen Teilen Änderungen auftreten können, so daß ein gewisser Zusammenhang von senilem Star mit Änderungen des Pupillarrandes und der hinteren Irisschicht erwartet werden kann.

Diese Erwartung trifft wirklich $z u$, wie die Beobachtungen Lindbergs ${ }^{1}$ ) zeigen, welcher in den Schlußsätzen seiner Doktorarbeit schreibt: „Es besteht ein Zusammenhang zwischen Cataracta subcapsularis senilis und Depigmentation und Defektbildung am Pupillarrande der hinteren Irisschicht. Dieser Zusammenhang beruht nicht allein auf einer gemeinschaftlichen Prädisposition durch höheres Alter. In der Mehrzähl der untersuchten Fälle von alten Personen, welche keine Linsentrübungen aufzeigten, würden auch die Pupillarrandveränderungen nicht wahrgenommen"s.

„,Die Fälle, in welchen die Diascleraldurchleuchtung wirklich brauchbare Resultate ergab, waren zu wenige, um einen sicheren SchluB zu ziehen über einen Parallelismus zwischen Depigmentation der hinteren Trisschicht und Starbildung. Die meisten dieser Fälle sprechen aber für einen ähnlichen Parallelismus." - Natürlich besteht absolut keine Gewißheit, daß dicse Änderungen eine Folge der fortwährenden Strahlenwirkung sind, aber möglich ist es, und sie reihen sich in unseren Gedankengang, ungezwungen ein. Daß Depigmentation der Iris durch ultraviolette Strahlen verursacht werden kann, sehen wir auch bei den Experimenten von Chotzen und Kuznitzky (s. w. u.).

Eine weitere Frage ist: Leidet die Netzhaut bei langjähriger $B \curvearrowright-$ strahlung mit Licht, welches viele ultraviolette Strahlen enthäl, ebenso wie die Ciliarfortsätze? Daß die Netzhaut durch diese Strahlen leiden kann, ist uns bekannt, bei Birch-Hirschfelds diesbezüglichen Experimenten traten bei akuter kräftiger Bestrahlung an der Netzhaut Degenerationen auf. Wir haben gesehen, daß Judson Daland es für wahrscheinlich erachtet, daß die bei den Eskimos so frequenten Retinochorioidaländerungen ultravioletten Strahlen zuzuschreiben seien.

Bei Schneeblindheit und Ophthalmia electrica treten ofter Netzhauterscheinungen auf, welche wahrscheinlioh ausgelöst werden von ultravioletten Strahlen höherer Wellenlänge in Verbindung mit Lichtstrahlen, wir sehen bei diesen Krankheiten eine Vorliebe für die Maculagegend, wie bei der Schneeblindheit Hänels, den'Zentralskotomen bei Kurzschluß, Flecke in der Maculagegend bei Bogenlampenbestrahlung, der helle Fleck in der Macula, welchen Lubinski beschreibt, das exzessive Zentralskotom, welches Heckel erwähnt, die Zentral-

1) J. C. Lindberg, Kliniska Undersökningar över Depigmenteringen av Pupillar randen och Genomlysbarheten av Tris. Akademisk Avhandling Helsingfors 1917 
skotome, welche Birch-Hirschfeld bei Blendung durch Quecksilberdampflampen konstatierte usw.

Die Bevorzugung der Macula ist kein Wunder, erstens bekommt doch der zentrale Teil der Netzhaut in den meisten Fällen die intensiyste Bestrahlung, dann ist nach Edingers Theorie die Macula der meist vulnerabele Teil, und überdies ist sie am mindesten geschützt. Nach. Birch-Hirsehfeld absorbieren die inneren Lagen der Netzhaut viel ultraviolette Strahlen, wodurch sie erkränken können; in der Macula aber sind die inneren Lagen nur wenige und sehr dünne, so daß das Netzhautepithel hier wenig geschützt ist und am leichtesten geschädigt werden kann. - Die Netzhaut verhält sich in dieser Beziehung wie der Ciliarkörper, sie leidet bei akuter, starker experimenteller Bestrahlung; bei Schneeblizdheit und durch artifizielle Lichtquellen.

Gibt es nun eine Netzhauterkrankung, welche wie der senile Star durch langjährige Bestrahlung mit den in Rede stehenden Strablen erklärt werden kann? Mir dünkt ja, nämlich die senile Maculadegeneration. Gewiß gibt es senile Maculadegenerationen, welche durch Gefäßsklerose verursacht werden, aber bei einer großen Zahl können wir Sklerose nicht feststellen. - Wenn dieses richtig ist, so wird also eine optisch sehr heterogene Linse die Macula vor seniler Degeneration schützen, aber selbe erkranken an seniler Trübung, während єine mehr homogene Linse klar bleiben wird, aber die Macula nicht schützt; es muß dann also ein gewisser Gegensatz bestehen zwischen senilem Star und seniler Maculadegeneration, wenn die eine da ist, wird wahrscheinlich die andere abwesend sein, während man bei bloßen senilen Abweichungen im Gegenteil erwarten müßte, daß sie sehr oft zusammen vorkommen.

In der Groninger Klinik trifft es tatsächlich zu, daß seniler Star und Maculadegeneration einander gewissermaßen ausschließen, wie ich in einer früheren Mitteilung dartat ${ }^{1}$ ).

Die von Halla uer u. a, aufgefundenen großen individuellen Unterschiede in Durchlässigkeit der Linse für Strahlen niedriger Wellenlänge lassen erwarten, daß das Auftreten von Star und Maculadegeneration sehr weehselnd sein kann.

1. Es wird Linsen geben, welche optisch so heterogen sind, daß sie die Netzhaut genügend schützen, ohne selber zu erkranken. Dieser Idealzustand kommt nur sehr selten vor.

2. Linsen, welche die Macula schützen, aber dabei via der Ciliarkörper sich selber in mehr oder geringerem Maße trüben, dies ist bei weitem die Mehrzahl; nach Hirschberg, v. HeB, Vogt u. a. bleiben nur sehr wenige Linsen im hohen Alter klar.

3. Linsen, welche die Macula nicht genügend schützen, aber selber klar bleiben,

1) Dieses Archiv 1918. 
4. Linsen, welche die Macula nicht genügend schützen und doch so viele Strahlen zerstreuen, daß durch Änderung des Ciliarepithels Trübungen auftreten.

Alle diese Kombinationen kommen in der Praxis tagtäglich vor.

Bei allen Ausführungen in dieser Beziehung muB man sich immer vergegenwärtigen, daß die hier besprochene Erkrankungsursache für Linse und Netzhaut, auch wenn sie wahrscheinlich ein Hauptfaktor für diese Abweichungen ist, doch immer nur einer der vielen Faktoren ist, welche Linsentrübungen und Netzhautdegeneration verursachen können.

Wenn dem so ist, so fragt es sich, ob es nötig ist, die Augen zu schützen und vor welchen Strahlen.

In casu werden dies hauptsächlich die ultravioletten Strahlen sein müssen, weil diese am meisten zerstreut werden und also das Ciliarepithel erreichen können. Muß man wegen der Möglichkeit, im hohen Alter Star oder Maculadegeneration zu bekommen, sein ganzes Leben Euphos-, Hallauer-, Crookes-, Fieuzal- oder dergleichen Gläser tragen? Mich dünkt nicht, die Beschwerden, fortwährend leichtgefärbte Gläser zu tragen, sind viel größer als die Gefahr einer eventuellen senilen $A b$ weichung; man wird aber gut, tun, wenn ungewöhnlich viel Strahlen das Auge erreichen, am Meeresstrand, bei Gletschertouren, auf stark reflektierenden Wegen u. dgl. Schutzbrillen zu tragen, welche vor allem die ultravioletten Strahlen abschwächen und nur, wenn die leuchtenden Strahlen Blendung verursachen, die genannten Gläser mit Grau verbinden, wie Euphosgrau, Hallauergrau usw.

Ist es für einen Emmetropen nicht wünschenswert, sich dauernd dureh die gelbgrünen Gläser vor dem Zuviel an Strahlen niedriger Wellenlänge zu schützen wegen dem Verdruß, Gläser zu tragen; ganz anders liegt die Sache für Leute, welche ohnehin schon verpflichtet sind, fortwährend Gläser zu tragen, wie Myope, Astigmate, hohe Hypermetrope usw. Bei diesen ist der Verdruß einer leichten Farbe der Gläser kleiner als die Gefahr, eventuell später senile Abweichungen zu bekommen. Die leicht gefärbten Gläser sind, wie wir von Schützen, Jägern, Fliegern u. a. wissen, dem Auge nicht unangenehm und setzen die Sehschärfe nicht herab; deswegen wird es, obwohl die gewöhnlichen Gläser an sich schon einen gewissen Schutz bieten, Empfehlung verdienen, die Refraktionsgläser von schwacher Hallauer- oder Euphosfarbe anzufertigen.

Für Lente, welche fortwährend bei intensiven artifiziellen Lichtquellen arbeiten müssen, ist es ratsam, die ultravioletten Strahlen abzuschwächen, entweder dureh Schutzbrillen oder besser durch Glasbirnen von einer der gelbgrünen Glassorten.

Es ist absolut nicht notwendig, von den Augen alle ultravioletten Strahlen fernzuhalten, denn der EinfluB, welcher erst in so vielen Dezennien die in Rede stehenden Krankheiten wahrscheinlich verursacht, 
braucht nur abgeschwächt, nicht ganz entfernt zu werden, um dem Auftreten der Abweichungen im späteren Leben vorzubeugen.

Es ist also nicht notwendig, wie schon vorgeschlagen ist, alle Lampengläser und Birnen, Fensterscheiben u. dgl. von vor Ultraviolett schützendem Glas zu machen, fortwährend Șchutzgläser zu tragen usw.; man braucht nur zu sorgen, daß die ultravioletten Strahlen nicht fortwährend auf das Auge einwirken, und kann dies tun in einer Weise, welche bei jedem Individuum den Verhältnissen angepaBt werden kann. Bis jetzt ist uns nichts bekannt über eventuellen Schaden, welcher dem Auge oder dem Körper durch Entziehung der ultravioletten Strahlen aus dem Lichte zugefügt werden könnte, "undenkbar ist dieses jedoch nicht. -

Bei therapeutischer Anwendung ultravioletten Lichtes wird man

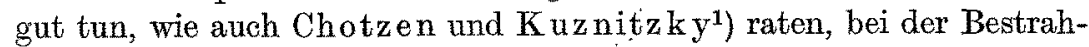
lung den unbeschützten Pupillarteil zu schonen. In dieser Weise vertahrend wird man bei den immerhin kurzdauernden Bestrahlungen das Auge voraussichtlich wenig oder nicht schädigen. Meines Erachtens wird man bei diesen kurzdauernden Bestrahlungen auch im Pupillarteil nicht viel Schaden erregen.

Die Ergebnisse meiner Experimente, Beobachtungen, Literaturbefunde und Erwägungen sind also:

1. Die Linse ist optisch heterogen und zerstreut demzufolge die ultravioletten Strahlen bedeutend.

2. Licht mit vielen ultravioletten Strahlen kann an Ciliarfortsätzen und Netzhaut Abweichungen verursachen.

3. Senile Linsentrübung und senile Maculadegeneration gehen in unserer Gegend nur selten zusammen, die Anwesenheit der einen Abweichung schließt gewissermäen die der anderen aus.

Wahrscheinlich ist, daß:

1. die Erkrankung der Ciliarfortsätze bei Bestrahlung dureh Licht mit vielen ultravioletten Strahlen verursacht wird durch diffuse Żerstreuung dieser Strablen in der Linse;

2. ein Hauptfaktor für die Entstehung des senilen Stars ist: Änderung der Ernährung der Linse infolge Erkrankung des Ciliarepithels, welche verursacht wird durch das fortwährend einwirkende Licht mit vielen ultravioletten Strahlen.

3. ein Hauptfaktor für die Entstehung der senilen Maculadegeneration ist der Einfluß von dem fortwährend einwirkenden Licht mit vielen ultravioletten Strahlen auf die Netzhaut.

1) Chotzen and Kuznitzky, Experimentelle und klinische Beiträge zur Bestrahlung der Cornea mit ultraviolettem Licht. Klin. Monatsbl. f. Augenheilk. 60, 198. 1918. 